\section{Low-dose versus high-dose fish oil for pain reduction and function improvement in patients with knee osteoarthritis}

Osteoarthritis (OA) is one of the leading generators of musculoskeletal pain and the main cause of disability. ${ }^{1}$ It has been considered an inflammatory disease with low grade inflammation affecting the synovium, cartilage and subchondral bone. ${ }^{2}$ To date, there is no disease-modifying OA drug. ${ }^{3}$ A few studies have evaluated the efficacy of fish oil in the treatment of OA; nevertheless, the effectiveness and precise benefits of fish oil intake in patients with OA are still far from well understood. We read with deep interest a recent article published in this journal by Hill et al, who found significant improvement of OA pain and function after treatment by fish oil, and suggested that the low-dose fish oil group had much better improvement in pain and function at 2 years in comparison to the high-dose one. ${ }^{5}$ The authors are congratulated for the excellent findings and we really appreciate the work performed by them; nevertheless, some worthwhile issues need further exploration.

First of all, this study was designed without a placebo treatment group. The placebo control is used to account for the placebo effect, and it is required in a large number of clinical trials. ${ }^{67}$ The authors explained that "It was considered unethical to prevent fish oil supplements for 2 years in these participants". 5 This is understandable. Nevertheless, since the efficacy of fish oil versus placebo in the treatment of knee OA is still unclear, we are not sure whether it is appropriate to conduct a clinical trial without placebo control to examine the anti-inflammatory efficacy of fish oil for knee OA, and to address that the pain scores in this study were 'comparable to those seen with placebo effect for pain'. 5

Second, the patient recruitment and completion of this study was not clearly described. The authors stated that some patients in both groups were withdrawn from therapy at Year 1 and Year 2, but not all these withdrawn patients were excluded from study assessment. ${ }^{8}$ Additionally, the sample size for analysis at Year 2 in the low-dose group was 85 after 3 patients were withdrawn from 90 patients at Year 1. However, the reasons for these issues were not addressed. These changes may have led to the bias of the results.

Third, the objective of the study was to compare the effects of an anti-inflammatory dose of fish oil with a lower dose of fish oil in knee OA. ${ }^{8}$ We fully agree with the authors that the OA joint inflammation can be partly reflected through the evaluation of bone marrow lesions by MRI, but we have no idea why the authors did not use MRI to assess synovitis, which is a hallmark of joint inflammation and closely related to joint pain and function in OA. ${ }^{9}$ In addition, the evaluation of proinflammatory cytokines which contribute to OA pathogenesis, such as interleukin $1 \beta$ and tumour necrosis factor (TNF), ${ }^{2}$ was not performed in the study. On the other hand, knee pain was selected as a parameter of OA inflammation in this study, but a substantial part of knee pain might result from other musculoskeletal diseases and the authors had not screened for these conditions. It was further noted that participants were provided with paracetamol tablets and were told that they could safely use up to eight per day. Is it possible that the patients with more severe joint pain have used more paracetamol tablets? Nevertheless, the authors then found that there was no difference between the two groups in the use of paracetamol or nonsteroidal antiinflammatory drugs (NSAIDs). The underlying mechanism would be interesting for further discussion.

Finally, the authors did not describe whether any other OA treatments were offered to the patients during the follow-up period, such as acupuncture, glucosamine, and intra-articular hyaluronan or steroid injection. Also high rates of serious adverse events (ie, non-elective hospital admissions) were found in both treatment groups and the detailed causes were missing. Some of the patients were reported to take knee surgery; it would be interesting to know more details of the surgery and whether the surgery had influenced the pain and the function assessment. Additionally, some other confounders may need to be addressed, such as the exercise type and intensity, occupation, alcohol-drinking status, smoking-status, diabetes mellitus, etc.

We respect the great contributions of the authors and we would also be very interested in the authors' response regarding the above issues.

\section{Yan Chen, ${ }^{1,2}$ Yong-Can Huang, ${ }^{1,3}$ William W Lu ${ }^{1,4}$}

${ }^{1}$ Department of Orthopaedics and Traumatology, The University of Hong Kong, Hong Kong SAR, P.R. China

${ }^{2}$ Department of Orthopaedics Trauma and Hand Surgery, The First Affiliated Hospital of Guangxi Medical University, Nanning, Guangxi, P.R. China

${ }^{3}$ Orthopedics Research Center, Peking University Shenzhen Hospital, Shenzhen, P.R. China

${ }^{4}$ Center for Human Tissue and Organs Degeneration, Shenzhen Institutes of Advanced Technology, CAS, China

Correspondence to Professor William W Lu, Ng Chun-Man Professorship in Orthopaedic Bioengineering, The University of Hong Kong, Room 907, Lab Block, 21 Sassoon Road, Hong Kong 999077, Hong Kong; wwlu@hku.hk

Contributors All the authors were involved in the study conception and manuscript design, manuscript drafting and revising, and final approval of the submitted version.

Funding The work was supported by Ng Chun-Man Foundation, National Science Foundation of China (NSFC 81270967), Shenzhen Peacock Project and the Research Grant Council of Hong Kong (HKU7149/13E).

Competing interests None declared.

Provenance and peer review Not commissioned; internally peer reviewed.

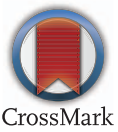

To cite Chen Y, Huang Y-C, Lu WW. Ann Rheum Dis 2016;75:e7.

Accepted 12 October 2015

Published Online First 9 November 2015

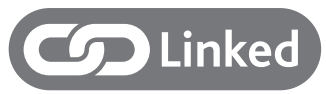

- http://dx.doi.org/10.1136/annrheumdis-2015-208781

Ann Rheum Dis 2016;75:e7. doi:10.1136/annrheumdis-2015-208754

REFERENCES

1 Zhang Y, Jordan JM. Epidemiology of osteoarthritis. Rheum Dis Clin North Am 2008;34:515-29.

2 Pelletier JP, Martel-Pelletier J, Abramson SB. Osteoarthritis, an inflammatory disease: potential implication for the selection of new therapeutic targets. Arthritis Rheum 2001;44:1237-47.

3 Bijlsma JW, Berenbaum F, Lafeber FP. Osteoarthritis: an update with relevance for clinical practice. Lancet 2011;377:2115-26.

4 Boe C, Vangsness CT. Fish oil and osteoarthritis: current evidence. Am J Orthop 2015;44:302-5.

5 Hill CL, March LM, Aitken D, et al. Fish oil in knee osteoarthritis: a randomised clinical trial of low dose versus high dose. Ann Rheum Dis 2016;75:3-9.

6 Bannuru RR, McAlindon TE, Sullivan MC, et al. Effectiveness and implications of alternative placebo treatments: a systematic review and network meta-analysis of osteoarthritis trials. Ann Intern Med 2015;163:365-72.

7 Hróbjartsson A, Gøtzsche PC. Is the placebo powerless? An analysis of clinical trials comparing placebo with no treatment. N Engl J Med 2001;344:1594-602.

8 Burnett WD, Kontulainen SA, McLennan CE, et al. Knee osteoarthritis patients with severe nocturnal pain have altered proximal tibial subchondral bone mineral density. Osteoarthritis Cartilage 2015:23:1483-90.

9 Roemer FW, Guermazi A, Javaid MK, et al. Change in MRI-detected subchondral bone marrow lesions is associated with cartilage loss: the MOST study. A longitudinal multicentre study of knee osteoarthritis. Ann Rheum Dis 2009;68:1461-5. 